\title{
Telmisartan delays myocardial fibrosis in rats with hypertensive left ventricular hypertrophy by TGF- $\beta 1 /$ Smad signal pathway
}

\begin{abstract}
Yong Zhang ${ }^{1,4}$, Liang Shao ${ }^{2,4}$, Aiqun $\mathrm{Ma}^{2}$, Gongchang Guan ${ }^{1}$, Junkui Wang ${ }^{1}$, Yaping Wang ${ }^{3}$ and Gang Tian ${ }^{2}$
Hypertensive myocardial remodeling has an important role in the pathophysiology of hypertensive disease. This study suggests that telmisartan (TEL) can inhibit myocardial fibrosis of hypertensive left ventricular hypertrophy (LVH) through the transforming growth factor- $\beta 1$ (TGF- $\beta 1$ )/Smad signaling pathway. Through echocardiography and hemodynamics, it was shown that TEL could improve cardiac function and reduce the degree of hypertensive LVH in hypertensive rats. Through immunoassay, it was shown that TEL could antagonize renin-angiotensin-aldosterone system expression in plasma and myocardial tissue. By Masson staining, Elisa and alkaline hydrolysis assays, it was demonstrated that TEL could significantly inhibit myocardial fibrosis in hypertensive rats and attenuate extracellular matrix-related proteins associated with pressure overload. Western blotting was used to detect the TGF- $\beta 1 / \mathrm{Smad}$ signaling pathway protein expression of myocardial tissue, and it was further found that TEL could inhibit activation of the TGF- $\beta 1 /$ Smad signaling pathway. In conclusion, TEL could inhibit myocardial local angiotensin II (Ang II) level by directly affecting the Ang II receptor. TEL may also restore the balance of matrix metalloproteinases/tissue inhibitor of metalloproteinases, reduce myocardial collagen fibrosis and delay hypertensive LVH by affecting the TGF- $\beta 1 / \mathrm{Smad}$ signaling pathway.
\end{abstract}

Hypertension Research (2014) 37, 43-49; doi:10.1038/hr.2013.119; published online 3 October 2013

Keywords: hypertensive myocardial remodeling; myocardial fibrosis; telmisartan; TGF- $\beta 1 /$ Smad

\section{INTRODUCTION}

Hypertensive myocardial remodeling is the pathophysiological basis of hypertensive diseases and eventually results in heart failure. Myocardial remodeling refers to changes in myocardial structure, function and phenotype that are caused by a series of complex molecular and cellular mechanisms and that represent the common pathological mechanism for the progression of the disease to congestive heart failure by a variety of causes. Myocardial remodeling manifests itself not only in myocardial cell apoptosis, necrosis, hypertrophy and extension, as well as cardiac hypertrophy, but also in the destruction of the dynamic balance between the myocardial extracellular matrix and fiber collagen fiber synthesis and degradation. ${ }^{1,2}$

The transforming growth factor- $\beta$ (TGF- $\beta$ ) family is a class of structurally related proteins that have a crucial role in extracellular matrix remodeling and growth, cell growth and apoptosis, intracellular homeostasis, tissue differentiation, morphogenesis, proliferation and migration., ${ }^{3,4}$ Smad proteins are the intracellular signaling pathway proteins of the TGF- $\beta$ superfamily, and they are associated with many cardiovascular diseases, such as myocardial fibrosis, heart failure, myocardial infarction, angioplasty trauma and pulmonary hypertension. They also participate in the development of the cardiac structure during the embryonic period. TGF- $\beta 1$ has an important role in extracellular matrix remodeling and myocardial fibrosis through downstream signal via Smad proteins. ${ }^{5,6}$ An increasing number of studies have confirmed that TGF- $\beta 1$ promotes the activity of matrix metalloproteinases (MMPs) in the myocardium and that TGF- $\beta 1$ upregulates the expressions of fibroblast MMP-2 and membrane-type MMP. $^{7}$ TGF- $\beta 1$ expression caused by norepinephrine can increase MMP-2 levels, ${ }^{8}$ and the elevated type I collagen induced by TGF- $\beta 1$ can also promote the activity of MMP-2. Further studies have confirmed that MMP-2 induced by TGF- $\beta 1$ can promote the migration and mobility of cardiac fibroblasts ${ }^{9}$ and cause extracellular matrix protein synthesis and myocardial fibrosis, a process that is closely connected to myocardial remodeling. Telmisartan (TEL) is part of a new generation of long-acting non-peptide angiotensin II (Ang II) receptor antagonists, and it selectively and irreversibly

${ }^{1}$ First Department of Cardiology, Shaanxi Provincial People's Hospital, Third Affiliated Hospital of Medical College of Xi'an Jiaotong University, Xi'an, Shaanxi, China; ${ }^{2}$ Department of Cardiovascular Medicine, First Affiliated Hospital of Medical College of Xi'an Jiaotong University, Xi'an, Shaanxi, China and ${ }^{3}$ Department of Age and Cardiology, Shaanxi Provincial People's Hospital, Third Affiliated Hospital of Medical College of Xi'an Jiaotong University, Xi'an, Shaanxi, China

${ }^{4}$ These authors contributed equally to this work.

Correspondence: Professor G Tian, Department of Cardiovascular Medicine, First Affiliated Hospital of Medical College of Xi'an Jiaotong University, No. 277 Yanta West Road, Xi'an, Shaanxi 710061, China.

E-mail: zhangyong.1@stu.xjtu.edu.cn

Received 31 March 2013; revised 10 June 2013; accepted 27 June 2013; published online 3 October 2013 
antagonizes the AT1 receptor of Ang II. Studies have shown that Ang II is the most important hormone that causes left ventricular hypertrophy $(\mathrm{LVH})$ and myocardial fibrosis. Studies have found that Ang II antagonists can inhibit the TGF- $\beta 1$ gene expression in rat myocardial and vascular tissues. Clinical studies have shown that angiotensin-converting enzyme inhibitors and Ang II receptor blockers decrease TGF- $\beta 1$ and the carboxy-terminal peptide of type I procollagen ${ }^{10-13}$ and reduce the synthesis of extracellular matrix proteins. The effects of TEL on TGF- $\beta 1$ or its downstream signals in rats with hypertension and LVH and TEL's effect on the rats' myocardial fibrosis have not been reported.

This study is intended to explore this aspect and provide a new theoretical basis for treatment strategies for hypertension-related myocardial remodeling. In this paper, we describe the pathway by which TEL affects myocardial fibrosis in rats.

\section{METHODS}

\section{Experimental animals and treatment}

Male SD rats (weight 180-230g) were purchased from the School of Medicine of Xi'an Jiaotong University, Xi'an, Shaanxi, China. In the experimental process, we strictly followed the relevant requirements of 'The Guidelines of Experimental Animals Care and Use' published by the American National Institutes of Health in 1996 and the Experimental Animals Management Regulations published by the State Science and Technology Commission of China in 2004.

Male SD rats were randomly divided into a sham group, a LVH group and a TEL group. In the TEL group, a pressure-overloaded LVH model was prepared by abdominal aorta constriction. Intragastric administration of TEL $10 \mathrm{mg} \mathrm{kg}^{-1} \mathrm{day}^{-1}$ was performed daily at noon for 2 weeks (in the 8-week group) and 4 weeks (in the 10-week group) from 6 weeks after surgery. Eight rats in which the induction of remodeling had been successful were identified in each group.

\section{The establishment of rat hypertension models}

Rats were fasted for $12 \mathrm{~h}$ before the operation, and the rats' body weight was measured. After full anesthesia through the intraperitoneal injection of $10 \%$ chloral hydrate (100-200 $\mathrm{mg} \mathrm{kg}^{-1}$ ), the abdominal aorta was exposed layer by layer. An 'L'-shaped blunt injection needle was placed along the blood vessel parallel to the abdominal aorta. The needle was withdrawn immediately to cause the abdominal aorta to form a fixed stenosis. Penicillin (400 $\left.000 \mathrm{U} \mathrm{ml}^{-1}\right)$ was dropped into the abdominal cavity, which was closed layer by layer. Then, intramuscular injections of penicillin were administered for three consecutive days to prevent infection. For the sham group, the ligation of the abdominal aorta was not conducted, but the remaining steps were the same as in the model group. Four weeks after remodeling surgery, the BL-420F biological and functional experimental system was used for the measurement of rat carotid arterial blood pressure; each anesthetized rat (10\% chloral hydrate) was measured three times consecutively, and the average value was taken. The criterion for the successful establishment of abdominal aortic coarctation in the hypertensive rat model was that systolic blood pressure differences between the surgery group and the sham group were $>50 \mathrm{~mm} \mathrm{Hg}$.

\section{The echocardiographic examination}

Ten percent chloral hydrate $\left(100-150 \mathrm{mg} \mathrm{kg}^{-1}\right)$ was used for full anesthesia of the test rats. A PHILIPS 7500 color Doppler ultrasound instrument (Philips, Amsterdam, Dutch) was applied to detect the indicators in the level short-axis view/section of the left ventricular papillary muscle. Three cardiac cycles were detected for each test rat, and then the average value was taken. End-diastolic interventricular septal thickness, end-diastolic left ventricular posterior wall thickness, left ventricular end-diastolic diameter, left ventricular ejection fraction and heart rate were measured and recorded.

\section{Hemodynamic monitoring}

Model rats were anesthetized using a $2 \%$ solution of sodium pentobarbital and then were fixed on an animal station. A catheter filled with heparinized saline was connected to the BL-420F biological and functional experimental system and inserted into the left ventricle via the right carotid artery. The required data were measured and recorded, including the left ventricular systolic pressure (LVSP), maximum rising rate of LVSP $(+\mathrm{dp} / \mathrm{dtmax})$ and maximum descent rate of LVSP (-dp/dtmax).

\section{The staining of rat left ventricular myocardial pathology}

Left ventricular myocardial specimens were taken from each group and sectioned transversely parallel to the coronary sulcus. Masson staining was used to observe the degree of myocardial fibrosis; myocardial cells were red and collagen was blue. Five sections were selected for each specimen, and four views were taken for each section (the selected views were away from the papillary muscle and perivascular collagen). The Image-Pro Plus software (Rockville, MD, USA) was used to calculate the myocardial collagen volume fraction.

Determination of rat metabolic-related matters of collagen fibers Arterial blood samples were collected and centrifuged at $3000 \times \mathrm{g}$ for $20 \mathrm{~min}$. The supernatant was collected and stored at $-80^{\circ} \mathrm{C}$. An ELISA kit was used to detect the concentrations of MMP-1, MMP-2, MMP-9, tissue inhibitor of metalloproteinase 1 (TIMP-1; American CUSBIO, Barksdale, DE, USA), procollagen type I carboxy-terminal peptide and type I collagen carboxyterminal cross-linked peptide (American R\&D, Muskogee, OK, USA) in the rat serum samples.

Myocardial hydroxyproline content was determined using alkaline hydrolysis. The oxidation products generated by hydroxyproline under the action of oxidants reacted with dimethylaminobenzaldehyde to form a purplish-red color. The myocardial hydroxyproline content could be calculated according to color depth.

Determination of rat Ang II and aldosterone (ALD) concentrations Arterial blood samples were collected and centrifuged at $3000 \times g$ for $20 \mathrm{~min}$. The supernatant was collected and stored at $-80^{\circ} \mathrm{C}$ for detection. Portions of the left ventricular muscle tissues were taken, and blood and flushing fluid were absorbed. These tissues were weighed, and $1 \mathrm{ml}$ physiological saline was added. The tissues were sufficiently ground and homogenized and then centrifuged at $4{ }^{\circ} \mathrm{C}$ for 3000 r.p.m. for $10 \mathrm{~min}$. The supernatant was collected and stored at $-70{ }^{\circ} \mathrm{C}$. The principle of competition mechanism was applied, and 125I-Ang II or 125I-Ald was added to produce a competitive immune response. The content of Ang II or ALD in the samples was determined.

\section{Detection by western blotting}

Myocardial protein expression was detected by western blotting. The bicinchonininc acid (BCA) method was used to detect the protein concentration. The sample volume was $50 \mu \mathrm{g}$, and $10 \%$ sodium dodecyl sulfate-polyacrylamide gel was used for separation and as a transmembrane. After blocking with 5\% nonfat milk for $2 \mathrm{~h}, 1: 1000$ rabbit anti-rat TGF- $\beta 1$ antibody (American R\&D), p-Smad2 antibody (Santa Cruz, Dallas, TX, USA), Smad6 antibody (CST, Danvers, MA, USA) and type I collagen antibody (ABCAM, Boston, MA, USA) were added, respectively. The mixture was incubated overnight at $4{ }^{\circ} \mathrm{C}$ and washed three times. The corresponding secondary antibody (goat anti-rabbit immunoglobulin G, Santa Cruz) was added and incubated at $37^{\circ} \mathrm{C}$ for $3 \mathrm{~h}$. After three washes, color development was conducted, and the film was developed after exposure. Western blotting bands of the target protein and internal reference $\beta$-actin were analyzed; the results were shown as the ratio of both integrated optical density (IOD), and statistical analysis was conducted.

\section{Statistical analysis}

All measurement data were presented as means \pm s.d., and the $t$-test was applied for the comparisons between two groups. For independent samples, the Mann-Whitney $U$ rank sum test was used for nonparametric tests. $P<0.05$ was considered to be statistically significant. 


\section{RESULTS}

\section{Basic data of $\mathrm{LVH}$ rat model}

At the sixth week following abdominal aortic coarctation, rat carotid artery blood pressure rose. Three measurements at different time points (with intervals of at least $4 \mathrm{~h}$ between two measurements) showed that the difference in the systolic blood pressure between the surgery group and the sham group was $>50 \mathrm{~mm} \mathrm{Hg}$. At the eighth and tenth weeks, the rat carotid artery pressure of the LVH group was recorded, and it remained stable at $148-160 \mathrm{~mm} \mathrm{Hg}$. At the same time, the rats' weights and left ventricular mass changes in the LVH group were recorded, and it was found that compared with the sham group, left ventricular mass increased significantly, and left ventricular mass fraction had a significant rise $(P<0.05)$ when body weight did not significantly change $(P>0.05)$. These results suggested that the hypertensive LVH rat model was successfully created. At the same time, the rats' basic data in the TEL group were recorded at the eighth and tenth weeks. It was found that at the eighth week, the rat carotid artery pressure of the TEL group showed a downward trend compared with that of the LVH group, but there was no significant difference $(P>0.05)$, nor were there significant changes in the data of

Table 1 Basic data of hypertensive LVH rats

\begin{tabular}{ccccc}
\hline & $A P(\mathrm{~mm} \mathrm{Hg})$ & BW $(g)$ & \multicolumn{1}{c}{ LVM (g) } & LVMI (mgg $\left.{ }^{-1}\right)$ \\
\hline Sham & & & & \\
$8 \mathrm{w}$ & $89.95 \pm 6.11$ & $451.7 \pm 22.7$ & 0.6830 .053 & $1.731 \pm 0.095$ \\
$10 \mathrm{w}$ & $94.28 \pm 6.04$ & $464.8 \pm 23.2$ & $0.674 \pm 0.054$ & $1.741 \pm 0.097$
\end{tabular}

$\angle V H$

$8 w \quad 154.57 \pm 6.54^{a} \quad 460.3 \pm 19.5 \quad 1.153 \pm 0.089^{a} \quad 2.956 \pm 0.223^{a}$

10 w $\quad 154.58 \pm 5.28^{\mathrm{a}} \quad 473.4 \pm 20.1 \quad 1.259 \pm 0.093^{\mathrm{a}} \quad 2.977 \pm 0.238^{\mathrm{a}}$

TEL

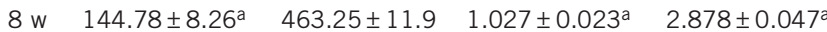

$10 w \quad 134.28 \pm 4.88^{a, b} \quad 446.75 \pm 12.3^{b} \quad 0.946 \pm 0.035^{a, b} \quad 2.118 \pm 0.037^{a, b}$

Abbreviations: AP, rat artery pressure; BW, body weight; $\mathrm{LVH}$, hypertensive left ventricular hypertrophy group; LVM, left ventricular mass; LVMI, left ventricular mass index; TEL,

telmisartan group; w, weeks.

$n=8$.

Compared with the sham group of the same period, $P<0.05$

${ }^{\mathrm{b}}$ Compared with LVH group of the same period, $P<0.05$. the other groups. However, at the tenth week, TEL had not only reduced the hypertension induced by aortic coarctation (although not to normal levels) but also reduced the body weight of the rats (possibly due to its diuretic effect) and reduced left ventricular mass (Table 1). There was a reduction in arterial pressure in the TEL group compared with the LVH group at 10 weeks, but this pressure was still significantly higher than that of the sham group. This phenomenon was likely due to the body weight reduction and alleviation of heart load; thus, it likely had little correlation with cardiac function improvement.

\section{Cardiac function changes in hypertensive left ventricular hypertrophic rats}

Compared with that of rats in the sham group at the same time, the left ventricular pressure overload of rats in the LVH group increased (LVSP increased), left ventricular remodeling progression increased (end-diastolic interventricular septal thickness, end-diastolic left ventricular posterior wall thickness and left ventricular end-diastolic diameter indicators increased), and diastolic function abnormalities started to appear $(-\mathrm{dp} / \mathrm{dtmax}$ decreased; $P<0.05)$. Meanwhile, compensatory increases appeared in the myocardial contractile function of rats in the LVH group, but there was no significant difference $(P>0.05)$. When TEL was given, it was found that TEL reduced the LVSP overload induced by abdominal aortic coarctation hypertension. TEL also partially reversed the left ventricular remodeling and corrected the diastolic function abnormalities caused by ventricular hypertrophy (compared with the LVH group during the same period, $P<0.05$ ). However, the hypertension was caused fundamentally by abdominal aortic coarctation, so TEL could not completely improve the cardiac function state and restore it to normal values (compared with the sham group on LVSP, -dP/dtmax, end-diastolic left ventricular posterior wall thickness, end-diastolic interventricular septal thickness and left ventricular end-diastolic diameter at the same period, $P<0.05$; Table 2).

\section{Effects of TEL on Ang II and ALD in hypertensive left ventricular hypertrophic rats}

We detected changes in Ang II and ALD expression in rat myocardium and plasma after abdominal aortic coarctation. The expressions of both Ang II and ALD in the myocardium and plasma of rats in the

Table 2 Cardiac function test of hypertensive LVH rats

\begin{tabular}{|c|c|c|c|c|c|c|c|c|}
\hline & $H R$ (b.p.m.) & LVSP (mm Hg) & $+d p / d t m a x\left(m m \mathrm{Hgs}^{-1}\right)$ & $-d p / d t m a x\left(m m H g s^{-1}\right)$ & $\operatorname{LVPWTd}(\mathrm{mm})$ & $\operatorname{IVSTd}(\mathrm{mm})$ & $\operatorname{LVDd}(\mathrm{mm})$ & $E F(\%)$ \\
\hline \multicolumn{9}{|l|}{ Sham } \\
\hline $8 w$ & $378 \pm 17$ & $112.9 \pm 3.46$ & $6008.12 \pm 427.28$ & $4553.44 \pm 269.90$ & $1.34 \pm 0.13$ & $1.21 \pm 0.08$ & $6.11 \pm 0.15$ & $74.88 \pm 3.68$ \\
\hline $10 w$ & $367 \pm 16$ & $117.9 \pm 3.97$ & $5917.95 \pm 411.33$ & $4463.22 \pm 239.20$ & $1.41 \pm 0.12$ & $1.32 \pm 0.09$ & $6.67 \pm 0.34$ & $75.08 \pm 6.79$ \\
\hline \multicolumn{9}{|l|}{$\mathrm{LVH}$} \\
\hline $8 w$ & $381 \pm 23$ & $159.4 \pm 2.73^{a}$ & $6269.08 \pm 207.21$ & $3316.66 \pm 178.98^{a}$ & $1.53 \pm 0.19^{a}$ & $1.49 \pm 0.12^{\mathrm{a}}$ & $6.59 \pm 0.23^{a}$ & $80.06 \pm 7.69$ \\
\hline $10 w$ & $378 \pm 22$ & $165.4 \pm 2.85^{\mathrm{a}}$ & $5809.70 \pm 198.88$ & $3200.78 \pm 159.44^{a}$ & $1.63 \pm 0.17^{a}$ & $1.51 \pm 0.13^{a}$ & $7.77 \pm 0.76^{a}$ & $78.05 \pm 4.38$ \\
\hline \multicolumn{9}{|l|}{$T E L$} \\
\hline $8 w$ & $366 \pm 27$ & $132.4 \pm 2.01^{\mathrm{a}, \mathrm{b}}$ & $5954.19 \pm 403.28$ & $3976.66 \pm 193.33^{a, b}$ & $1.45 \pm 0.14^{a, b}$ & $1.45 \pm 0.09^{a}$ & $6.28 \pm 0.52^{a, b}$ & $77.96 \pm 8.14$ \\
\hline $10 w$ & $354 \pm 25$ & $126.4 \pm 2.23^{a, b}$ & $6134.26 \pm 212.37$ & $4115.76 \pm 189.66^{a, b}$ & $1.39 \pm 0.16^{a, b}$ & $1.46 \pm 0.11^{\mathrm{a}}$ & $7.00 \pm 0.50^{a, b}$ & $84.96 \pm 5.32$ \\
\hline
\end{tabular}

Abbreviations: + dp/dtmax, maximum rising rate of LVSP; -dp/dtmax, maximum descent rate of LVSP; EF, left ventricular ejection fraction; HR, heart rate; IVSTd, end-diastolic interventricular septal thickness; LVDd, left ventricular end-diastolic diameter; LVH, hypertensive left ventricular hypertrophy group; LVSP, left ventricular systolic pressure; LVPWTd, end-diastolic left ventricular posterior wall thickness; TEL, telmisartan group; w, weeks.

$n=8$.

Compared with the sham group of the same period, $P<0.05$

${ }^{\mathrm{b}}$ Compared with LVH group of the same period, $P<0.05$. 
Table 3 Determination of angiotensin II and ALD expressions of hypertensive $\mathrm{LVH}$ rats

\begin{tabular}{|c|c|c|c|c|}
\hline & \multicolumn{2}{|c|}{ Serum } & \multicolumn{2}{|c|}{ Tissue } \\
\hline & Ang $/ /\left(p g \mathrm{~m}^{-1}\right)$ & $A L D\left(p g m l^{-1}\right)$ & Ang $/ /\left(p g m r^{-1}\right)$ & $A L D\left(p g \mathrm{ml}^{-1}\right)$ \\
\hline \multicolumn{5}{|l|}{ Sham } \\
\hline $8 w$ & $155.88 \pm 58.76$ & $694.09 \pm 46.43$ & $60.26 \pm 7.87$ & $42.08 \pm 10.03$ \\
\hline $10 \mathrm{w}$ & $145.94 \pm 61.15$ & $700.03 \pm 40.13$ & $67.86 \pm 7.26$ & $39.07 \pm 9.67$ \\
\hline \multicolumn{5}{|l|}{$\mathrm{LVH}$} \\
\hline $8 w$ & $392.65 \pm 41.67^{a}$ & $946.73 \pm 86.69^{a}$ & $136.86 \pm 13.01^{a}$ & $75.34 \pm 11.75^{a}$ \\
\hline $10 w$ & $412.65 \pm 44.23^{a}$ & $976.55 \pm 90.66^{a}$ & $145.77 \pm 14.41^{a}$ & $79.65 \pm 10.65^{\circ}$ \\
\hline \multicolumn{5}{|l|}{ TEL } \\
\hline $8 w$ & $457.56 \pm 58.33^{a}$ & $789.07 \pm 63.22^{\mathrm{a}, \mathrm{b}}$ & $77.76 \pm 13.22^{b}$ & $46.34 \pm 6.65^{b}$ \\
\hline $10 w$ & $487.95 \pm 61.41^{a}$ & $710.06 \pm 63.23^{b}$ & $83.95 \pm 10.44^{b}$ & $42.22 \pm 5.35^{b}$ \\
\hline
\end{tabular}

Abbreviations: Ang II, angiotensin II; ALD, aldosterone; LVH, hypertensive left ventricular hypertrophy group; TEL, telmisartan group; w, weeks.

$n=8$.

Compared with the sham group of the same period, $P<0.05$

${ }^{b}$ Compared with LVH group of the same period, $P<0.05$.

LVH group increased significantly compared with that of the sham group in the same period $(P<0.05)$. The antagonistic role of TEL in the renin-angiotensin-aldosterone system (RAAS) was very significant, and compared with that of the LVH group of the same period, the ALD expression in plasma and myocardium in the TEL group significantly decreased because of the inhibition of the Ang II AT1 receptor $(P<0.05)$. However, Ang II expression in myocardium and plasma was inconsistent. Compared with that of the LVH group of the same period, Ang II expression in plasma further increased $(P<0.05)$, whereas Ang II expression in myocardium decreased significantly $(P<0.05$; Table 3$)$.

\section{TEL alleviated myocardial fibrosis in hypertensive left ventricular} hypertrophic rats

The degree of myocardial fibrosis in the hypertensive left ventricular hypertrophic rats could be identified through Masson staining (Figure 1a). Normal myocardial tissue was red, and the blue fibrin component appeared in the myocardial interstitium after abdominal aortic coarctation. Myocardial interstitial fibrosis could limit myocardial diastolic function. The Image-Pro Plus software was used to calculate the volume fraction of myocardial collagen (specific method as previously described). The collagen volume fraction of the $\mathrm{LVH}$ group increased significantly compared with that of the sham group in the same period (LVH: sham $=(12.06 \pm 1.32):(2.12 \pm 0.23)$, 8 weeks; LVH: sham $=(12.65 \pm 1.41):(2.34 \pm 0.18), 10$ weeks; $n=5, P<0.05)$. However, TEL significantly reduced the degree of myocardial fibrosis in hypertensive left ventricular hypertrophic rats $(\mathrm{TEL}: \quad \mathrm{LVH}=(9.73 \pm 1.16):(12.06 \pm 1.32), \quad 8$ weeks; TEL: $\mathrm{LVH}=(7.83 \pm 2.06):(12.06 \pm 1.32), \quad 10 \quad$ weeks; $n=5, \quad P<0.05 ;$ Figure $1 b)$.

\section{The effect of TEL on metabolic-related matters of collagen fibers in} hypertensive left ventricular hypertrophic rats

After further analysis of TEL's effects on the metabolic-related matters of myocardial fibrosis collagen in hypertensive left ventricular hypertrophic rats, it was found that compared with that of the sham group for the same period, the serum MMP-1 (mainly degrading type I and type III collagen) of rats in the LVH group decreased significantly $(P<0.05)$, whereas serum MMP-2 and MMP-9 (mainly degrading denatured type I and type III collagen) and serum TIMP-1 (feedback inhibiting the activity of MMP-1 and MMP-9) increased significantly $(P<0.05)$. Serum procollagen type I carboxy-terminal peptide (reflecting type I collagen synthesis) increased significantly $(P<0.05)$ and serum type I collagen carboxy-terminal cross-linked peptide (reflecting type I collagen degradation) had no significant change $(P>0.05)$. Meanwhile, myocardial hydroxyproline reflecting total collagen metabolism increased significantly $(P<0.05)$. This finding suggested that the collagen metabolic imbalance in hypertensive LVH rats occurred, synthesis increased and degradation decreased, and the degradation was mainly the degradation of denatured collagen (Table 4).

In the TEL group, TEL had different effects on the metabolicrelated matters of myocardial fibrosis collagen of hypertensive left ventricular hypertrophic rats compared with the LVH group for the same period. TEL could not reduce serum MMP-9 $(P>0.05)$, but it could significantly reduce serum MMP-2 expression close to the normal level $(P<0.05)$. At the same time, it was found, in our study, that compared with that of the LVH group for the same period, serum MMP-1 expression in the TEL group increased significantly $(P<0.05)$. TEL's effect on TIMP-1, procollagen type I carboxyterminal peptide and type I collagen carboxy-terminal cross-linked peptide was weaker, and although there was a significant difference in expression compared with that of the LVH group $(P<0.05)$, it did not reach the level of the normal group $(P<0.05)$. After further detection of TEL's effect on myocardial total collagen metabolism, it was found that hydroxyproline in the TEL group decreased significantly compared with that of the LVH group for the same period $(P<0.05)$. These results suggested that TEL could correct MMPs/ TIMP imbalance in hypertensive left ventricular hypertrophic rats, reduce rat collagen synthesis and promote collagen decomposition, thereby reducing the myocardial fibrosis of hypertensive $\mathrm{LVH}$ rats (Table 4).

\section{The effect of TEL on the signaling pathway of TGF- $\beta 1 / \mathrm{Smad}$ of} myocardial fibrosis

We further analyzed through which signaling pathway TEL affected myocardial collagen metabolism in hypertensive left ventricular hypertrophic rats. At the eighth week, the myocardial collagen I protein of the rats in the LVH group significantly increased compared with that of the sham group $(P<0.05)$, being up to two times higher. Meanwhile, the expression of the key protein TGF- $\beta 1$ in collagen fiber metabolism and the expression of its downstream signaling proteins P-Smad2 and Smad6 increased significantly $(P<0.05)$, and in particular, the increases in TGF- $\beta 1$ and P-Smad were up to 20 -fold and 40 -fold, respectively. The expressions of myocardial TGF- $\beta 1$ and $\mathrm{P}$-Smad of the rats in the TEL group decreased significantly and were only half of those of the LVH group. However, at the eighth week, the expressions of Smad6 and collagen I protein in the rats in the TEL group had no significant difference compared with those of the $\mathrm{LVH}$ group $(P>0.05$; Figure 2a).

At the tenth week, the TGF- $\beta 1$ and P-Smad proteins of the rats in the LVH group still had significant differences compared with those of the sham group $(P<0.05)$. Moreover, the expressions of myocardial Smad6 and collagen I protein in the LVH group also showed a greater differences compared with those of the sham group, and the differences expanded up to 18 -fold and 10-fold, respectively. The expressions of the myocardial TGF- $\beta /$ Smad signaling pathway protein and collagen I protein of the rats in the TEL group decreased significantly compared with that of the LVH group for the same period $(P<0.05)$. The expressions of myocardial TGF- $\beta 1$, Smad6 and 
a

$8 w$
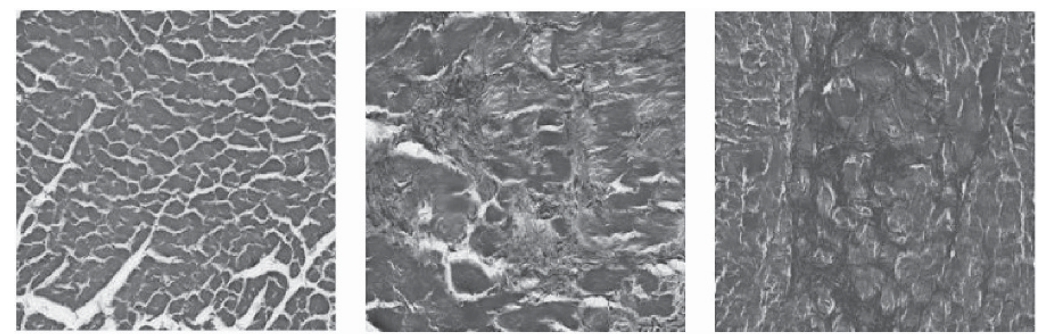

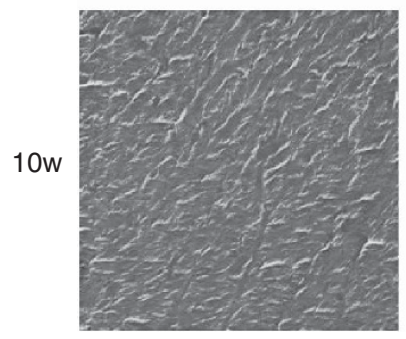

Sham

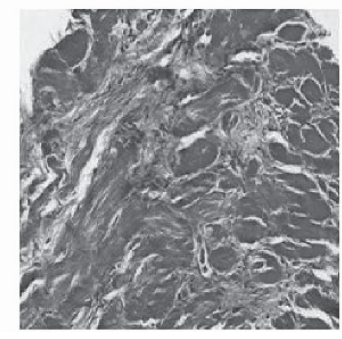

LVH

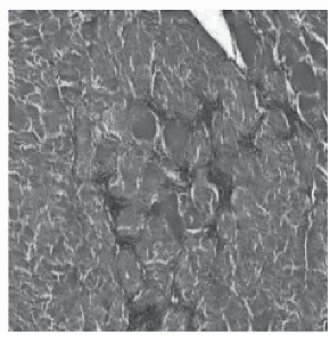

TEL

b

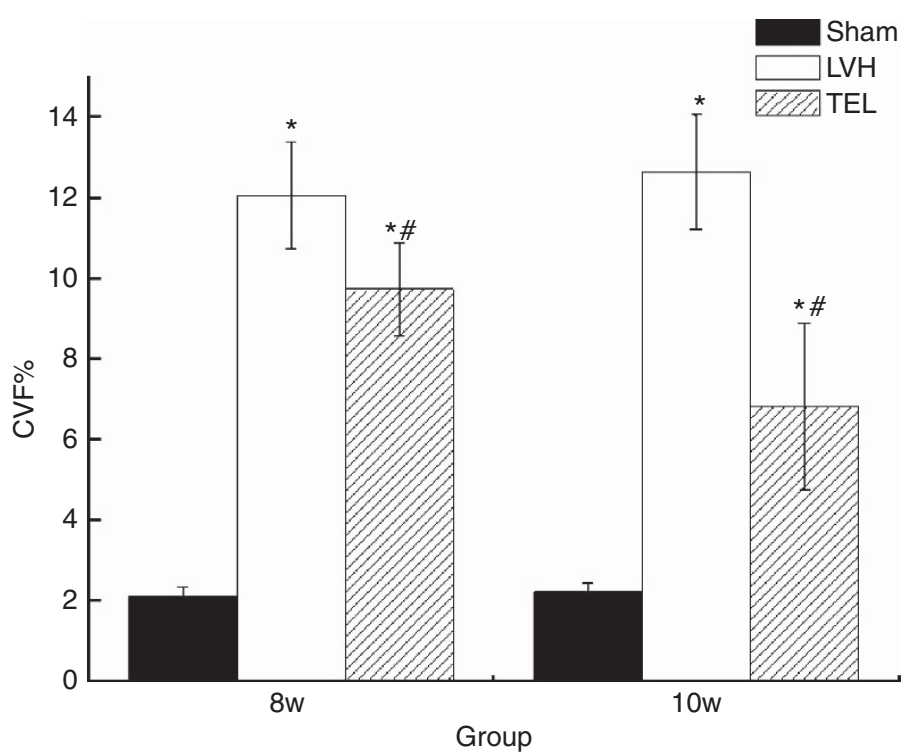

Figure 1 (a) Myocardial Masson staining of hypertensive LVH rats. Normal myocardium shown in red and cellulose shown in blue ( $\times 400)$. (b) Comparison of collagen volume fraction of hypertensive LVH rats. $n=5$. ${ }^{*}$ Compared with the sham group of the same period, $P<0.05$. \#Compared with LVH group of the same period, $P<0.05$. CVF, collagen volume fraction; LVH, left ventricular hypertrophy; TEL, telmisartan; w, weeks. A full color version of this figure is available at the Hypertension Research journal online

collagen I of rats in the TEL group were close to normal levels $(P>0.05)$, whereas P-Smad2 protein expression increased to abnormally high levels compared with the level of the sham group $(P<0.05)$ but decreased significantly compared with the level of the LVH group $(P<0.05)$.

\section{DISCUSSION}

This study demonstrated that the abdominal aortic coarctation of rats could cause hypertensive LVH and myocardial interstitial fibrosis. ${ }^{14}$ However, TEL could reduce the degree of myocardial fibrosis and thus improve cardiac function by inhibiting TGF- $\beta 1 / \mathrm{Smad}$ signaling pathway protein expression.

Left ventricular compensatory hypertrophy and cardiac diastolic dysfunction could develop and result in changes in the left ventricular structure in abdominal aortic coarctation hypertension models. Eventually, it could result in cardiac diastolic dysfunction and even heart failure. In hypertensive left ventricular hypertrophic rats, both the TGF- $\beta 1 /$ Smad signaling pathway and the RAAS are activated in circulation and myocardial tissue. The MMP-1 level decreases significantly, and the TIMP-1 level increases significantly, ${ }^{15-17}$ whereas the myocardial collagen synthesis increases $^{18}$ and degradation decreases. TGF- $\beta 1 / \mathrm{Smad}$ signaling pathway is closely related with myocardial fibrosis and interacts with the RAAS, and then affects the balance of MMPs/TIMPs, thus causing myocardial fibrosis.

In the circulatory system, almost all of the physiological effects of Ang II were mediated by activating the Ang II type 1 receptor. ${ }^{19}$ The major role of RAAS was to directly promote systemic arteriole contraction to high blood pressure and to promote venous contraction to increased returned blood volume. In addition, Ang II could produce and release ALD, which could increase the reabsorption of $\mathrm{Na}+$ in renal tubules. The local RAAS in heart is different from the role of systemic RAAS. ${ }^{20}$ The cardiac 
Table 4 Determination of collagen metabolic-related matters of hypertensive LVH rats

\begin{tabular}{|c|c|c|c|c|c|c|c|}
\hline & $M M P-1\left(p g m l^{-1}\right)$ & MMP-2 $\left(n g m l^{-1}\right)$ & $M M P-9\left(p g m l^{-1}\right)$ & $T I M P-1\left(n g m l^{-1}\right)$ & $P I C P\left(n g m l^{-1}\right)$ & ICTP $\left(n g m r^{-1}\right)$ & $\operatorname{HYP}\left(\mu g g^{-1}\right)$ \\
\hline \multicolumn{8}{|l|}{ Sham } \\
\hline $8 w$ & $42.64 \pm 9.53$ & $12.01 \pm 2.24$ & $243.52 \pm 13.61$ & $3.79 \pm 1.53$ & $17.63 \pm 1.01$ & $3.96 \pm 0.81$ & $158.12 \pm 22.32$ \\
\hline \multicolumn{8}{|l|}{$L V H$} \\
\hline $8 w$ & $28.09 \pm 4.97^{a}$ & $15.04 \pm 2.58^{a}$ & $336.90 \pm 15.55^{a}$ & $9.06 \pm 3.21^{\mathrm{a}}$ & $30.77 \pm 8.54^{a}$ & $4.23 \pm 0.77$ & $601.33 \pm 36.06^{a}$ \\
\hline \multicolumn{8}{|l|}{ TEL } \\
\hline $8 w$ & $38.22 \pm 7.88^{b}$ & $13.52 \pm 1.73^{b}$ & $314.56 \pm 21.09^{a}$ & $6.60 \pm 1.91^{\mathrm{a}, \mathrm{b}}$ & $22.94 \pm 1.96^{a, b}$ & $4.12 \pm 0.61$ & $462.38 \pm 47.60^{a, b}$ \\
\hline $10 w$ & $40.86 \pm 7.12^{b}$ & $11.58 \pm 1.93^{b}$ & $300.65 \pm 22.49^{a}$ & $5.50 \pm 2.10^{a, b}$ & $24.24 \pm 2.26^{a, b}$ & $3.90 \pm 0.74$ & $373.38 \pm 50.90^{a, b}$ \\
\hline
\end{tabular}

Abbreviations: HYP, hydroxyproline; ICTP, type I collagen carboxy-terminal cross-linked peptide; LVH, hypertensive left ventricular hypertrophy group; MMP, matrix metalloproteinase; PICP, procollagen type I carboxy-terminal peptide; TEL, telmisartan group; TIMP, tissue inhibitor of metalloproteinase; w, weeks.

$n=8$.

${ }^{a}$ Compared with the sham group of the same period, $P<0.05$.

${ }^{\mathrm{b}} \mathrm{Compared}$ with LVH group of the same period, $P<0.05$.

a

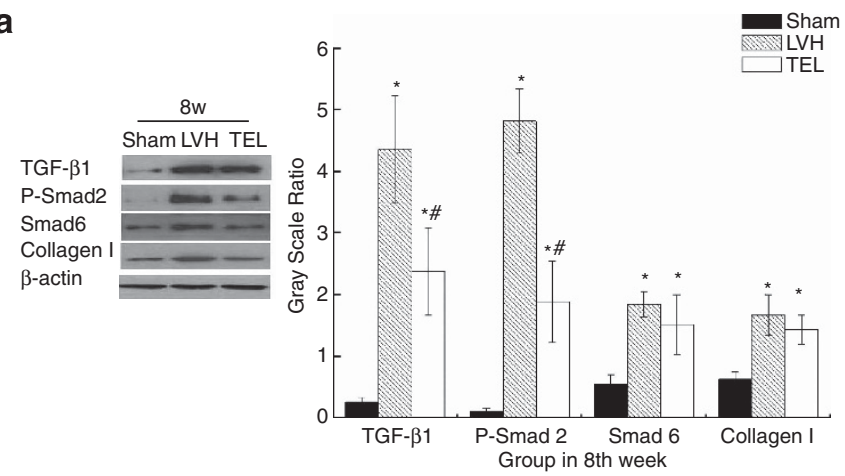

b

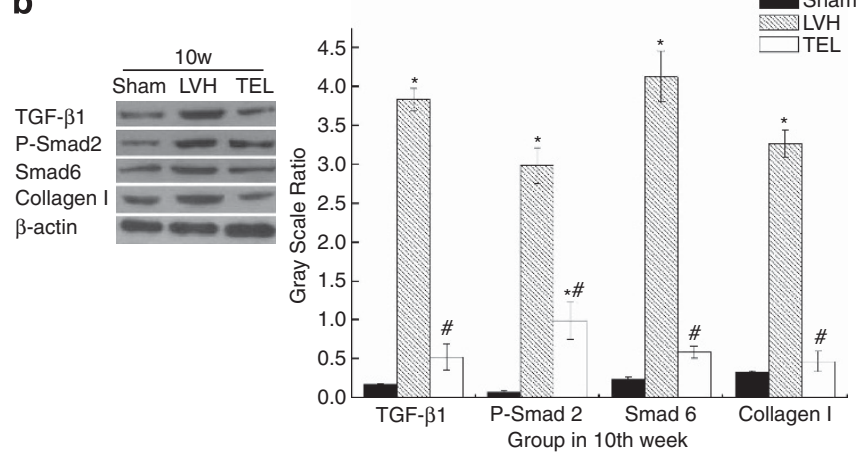

Figure 2 Transforming growth factor- $\beta 1$ (TGF- $\beta 1) / S m a d$ signaling pathway protein expression changes of hypertensive left ventricular hypertrophy (LVH) rats. (a) At eighth week, telmisartan (TEL) could significantly reduce myocardial TGF- $\beta 1$ and P-Smad2 protein expression of hypertensive LVH rats. (b) At tenth week, TEL could not only reduce myocardial TGF- $\beta 1$ and P-Smad2 protein expression of hypertensive LVH rats, but also significantly reduce Smad collagen I protein expression. $n=8$. ${ }^{*}$ Compared with the sham group of the same period, $P<0.05$. " Compared with LVH group of the same period, $P<0.05$.

RAAS could strengthen positive inotropic effects, induce cardiac hypertrophy, regulate coronary resistance and inhibit cardiomyocyte growth.

The binding of TGF- $\beta 1$ and its receptor causes Smad2 phosphorylation, and P-Smad2 has a subsequent role. Only P-Smad2 in Smad2/3 is active, so P-Smad 2 could be used as an indicator of TGF- $\beta$ signaling activity. $^{21}$ On the contrary, the Smad6 and bone morphogenetic protein receptor 1 combination could prevent Smad2 phosphorylation and lead to the disintegration of the Smad2 complexes. Smad6 also inhibits smooth muscle cell growth and maintains vascular smooth muscle cell phenotypes, thereby preventing damage to the vasculature after vascular proliferative disorders. ${ }^{22}$

TEL, as a new generation of long-acting non-peptide Ang II receptor antagonists, selectively and irreversibly antagonizes the AT1 receptor and can block the effects caused by RAAS-induced elevated Ang II. RAAS activation may affect MMP and TIMP transcription and expression levels and destroy the balance of MMPs/TIMPs by influencing myocardial tissue and myocardial cell TGF- $\beta$ 1/Smad signaling pathway. ${ }^{23}$ Thereby, it makes collagen synthesis increase and collagen degradation decrease, and results in myocardial fibrosis. TEL can inhibit the activation of the TGF- $\beta 1 /$ Smad signaling pathway, thus affecting the expressions of MMP-1, MMP-2, MMP-9 and TIMP- ${ }^{24}$ to restore the balance of MMPs/TIMPs, improve myocardial collagen metabolism and alleviate hypertensive LVH.

Notably, in this model, hypertensive LVH was induced by abdominal aortic coarctation, so TEL could not eradicate the etiology of hypertension or fundamentally curb the trend of left ventricular remodeling and myocardial interstitial fibrosis. Lowing arterial pressure could alleviate hypertensive LVH and myocardial interstitial fibrosis. This has been proven in a number of experimental models. ${ }^{25,26}$ However, TEL cannot eradicate the etiology of hypertension, which was induced by abdominal aortic coarctation, so the arterial pressure is still significantly higher in the TEL group than in the sham group in our research at 10 weeks $(P<0.05$; Table 1). The stimulating factor of Smad signaling is still efficacious at 10 weeks. In addition, the arterial pressure-lowering effects of TEL are not significantly different compared with the LVH group at 8 weeks, but there is still a decrease in Smad signaling and collagen metabolism-related matters and an improvement in cardiac function. Therefore, there should be another independent pressure-lowering way to inhibit myocardial fibrosis of hypertensive LVH rats, which is the TGF- $\beta 1 /$ Smad signaling pathway in our study in summary. This once again confirmed that combined with TEL in the clinical treatment of the primary disease of hypertension, myocardial remodeling could be delayed, myocardial interstitial fibrosis was reduced and thus cardiac function was improved. 
In summary, this experiment reveals that TEL does not depend only on the mechanism of inhibiting myocardial fibrosis by reducing blood pressure. When TEL cannot eradicate the etiology of hypertension, it can inhibit the myocardial fibrosis of hypertensive left ventricular hypertrophic rats through the TGF- $\beta 1 / \mathrm{Smad}$ signaling pathway, reduce the LVSP load of hypertensive rats and delay left ventricular remodeling, and eventually improve cardiac function.

\section{CONFLICT OF INTEREST}

The authors declare no conflict of interest.

\section{ACKNOWLEDGEMENTS}

The authors disclosed receipt of the following financial support for the research, authorship, and/or publication of this article: supported by Shaanxi Provincial Natural Science Basic Research Program (no. 2013JM4041) to Yong Zhang.

1 Olsen MH, Christensen MK, Wachtell K, Tuxen C, Fossum E, Bang LE, Wiinberg N, Devereux RB, Kjeldsen SE, Hildebrandt P, Dige-Petersen H, Rokkedal J, Ibsen H. Markers of collagen synthesis is related to blood pressure and vascular hypertrophy: a LIFE substudy. J Hum Hypertens 2005; 19: 301-307.

2 López B, González A, Querejeta R, Larman M, Díez J. Alterations in the pattern of collagen deposition may contribute to the deterioration of systolic function in hypertensive patients with heart failure. J Am Coll Cardiol 2006; 48: 89-96.

3 Chen YG, Meng AM. Negative regulation of TGF-beta signaling in development. Cell Res 2004; 14: 441-449.

4 Massague J, Gomis RR. The logic of TGF beta signaling. FEBS Lett 2006; 580 : 2811-2820.

5 Zhu XY, Daghini E, Rodriguez-Porcel M, Chade AR, Napoli C, Lerman A, Lerman LO. Redox-sensitive myocardial remodeling and dysfunction in swine diet-induced experimental hypercholesterolemia. Atherosclerosis 2007; 193: 62-69.

6 Xia Y, Lee K, Li N, Corbett D, Mendoza L, Frangogiannis NG. Characterization of the inflammatory and fibrotic response in a mouse model of cardiac pressure overload. Histochem Cell Biol 2009; 131: 471-481.

7 Hein S, Arnon E, Kostin S, Schönburg M, Elsässer A, Polyakova V, Bauer EP, Klövekorn WP, Schaper J. Progression from compensated hypertrophy to failure in the pressure-overloaded human heart - structural deterioration and compensatory mechanisms. Circulation 2003; 107: 984-991.

8 Briest W, Homagk L, Rassler B, Ziegelhöffer-Mihalovicová B, Meier H, Tannapfel A, Leiblein S, Saalbach A, Deten A. Zimmer HG. Norepinephrine-induced changes in cardiac transforming growth factor-beta isoform expression pattern of female and male rats. Hypertension 2004; 44: 410-418.

9 Stawowy P, Margeta C, Kallisch H, Seidah NG, Chrétien M, Fleck E, Graf K. Regulation of matrix metalloproteinase MT1-MMP/MMP-2 in cardiac fibroblasts by TGF-beta 1 involves furin-convertase. Cardiovasc Res 2004; 63: 87-97.

10 Müller-Brunotte R, Kahan T, López B, Edner M, González A, Díez J, Malmqvist K. Myocardial fibrosis and diastolic dysfunction in patients with hypertension: results from the Swedish Irbesartan Left Ventricular Hypertrophy Investigation versus Atenolol (SILVHIA). J Hypertens 2007; 25: 1958-1966.

11 Ciulla MM, Paliotti $R$, Esposito A, Diez J, López B, Dahlöf $B$, Nicholls MG, Smith RD, Gilles L, Magrini F, Zanchetti A. Different effects of antihypertensive therapies based on losartan or atenolol on ultrasound and biochemical markers of myocardial fibrosis - results of a randomized trial. Circulation 2004; 110: 552-557.

12 Kawasaki D, Kosugi K, Waki H, Yamamoto K, Tsujino T, Masuyama T. Role of activated renin-angiotensin system in myocardial fibrosis and left ventricular diastolic dysfunction in diabetic patients - reversal by chronic angiotensin II type 1A receptor blockade. Circ J 2007; 71: 524-529.

13 Kawano H, Toda G, Nakamizo R, Koide Y, Seto S, Yano K. Valsartan decreases type I collagen synthesis in patients with hypertrophic cardiomyopathy. Circ J 2005; 69: 1244-1248.

14 Cuspidi C, Sala C, Zanchetti A. Management of hypertension in patients with left ventricular hypertroph. Curr Hypertens Rep 2007; 9: 498-505.

15 Iraqi W, Rossignol P, Angioi M, Fay R, Nuée J, Ketelslegers JM, Vincent J, Pitt B, Zannad $\mathrm{F}$. Extracellular cardiac matrix biomarkers in patients with acute myocardial infarction complicated by left ventricular dysfunction and heart failure insights from the Eplerenone Post-Acute Myocardial Infarction Heart Failure Efficacy and Survival Study (EPHESUS) Study. Circulation 2009; 119: 2471-2479.

16 Zhao W, Zhao T, Chen Y, Ahokas RA, Sun Y. Oxidative stress mediates cardiac fibrosis by enhancing transforming growth factor-betal in hypertensive rats. Mol Cell Biochem 2008; 317: 43-50.

17 Yuan WH, Varga J. Transforming growth factor-beta repression of matrix metalloproteinase-1 in dermal fibroblasts involves Smad3. J Biol Chem 2001; 276: 38502-38510.

18 Li P, Wang D, Lucas J, Oparil S, Xing D, Cao X, Novak L, Renfrow MB, Chen YF. Atrial natriuretic peptide inhibits transforming growth factor beta-induced Smad signaling and myofibroblast transformation in mouse cardiac fibroblasts. Circ Res 2008; 102: $185-192$.

19 D'Amore A, Black MJ, Thomas WG. The angiotensin II type 2 receptor causes constitutive growth of cardiomyocytes and does not antagonize angiotensin II type 1 receptor-mediated hypertrophy. Hypertension 2005; 46: 1347-1354.

20 Donnelly R, Collinson DJ, Manning G. Hypertension, matrix metalloproteinases and target organ damage. J Hypertens 2003; 21: 1627-1630.

21 Khan R, Sheppard R. Fibrosis in heart disease: understanding the role of transforming growth factor-beta in cardiomyopathy, valvular disease and arrhythmia. Immunology 2006; 118: 10-24.

22 Leppäranta O, Myllärniemi M, Salmenkivi K, Kinnula VL, Keski-Oja J, Koli K. Reduced phosphorylation of the TGF-Beta signal transducer Smad2 in emphysematous human lung. COPD 2009; 6: 234-241.

23 Dasgupta C, Zhang L. Angiotensin II receptors and drug discovery in cardiovascular disease. Drug Discov Today 2011; 16: 22-34.

24 Tziakas DN, Chalikias GK, Stakos DA, Papazoglou D, Papanas N, Papatheodorou K, Chatzikyriakou SV, Kotsiou S, Maltezos E, Boudoulas H. Effect of angiotensin converting enzyme insertion/deletion genotype on collagen type I synthesis and degradation in patients with atrial fibrillation and arterial hypertension. Expert Opin Pharmacother 2007; 8: 2225-2234.

25 Okada M, Kosaka N, Hoshino Y, Yamawaki H, Hara Y. Effects of captopril and telmisartan on matrix metalloproteinase-2 and -9 expressions and development of left ventricular fibrosis induced by isoprenaline in rats. Biol Pharm Bull 2010; 33 : 1517-1521.

26 Liang B, Leenen FH. Prevention of salt-induced hypertension and fibrosis by AT1-receptor blockers in Dahl S rats. J Cardiovasc Pharmacol 2008; 51: 457-466. 